\title{
Mineral Deposition in the Extracellular Matrices of Vertebrate Tissues: Identification of Possible Apatite Nucleation Sites on Type I Collagen
}

\author{
William J. Landis ${ }^{\text {a }}$ Frederick H. Silver ${ }^{\text {b }}$ \\ a Department of Integrative Medical Sciences, Northeastern Ohio Universities Colleges of Medicine and Pharmacy, \\ Rootstown, Ohio, and ${ }^{\mathrm{b}}$ Department of Pathology and Laboratory Medicine, Robert Wood Johnson Medical School, \\ University of Medicine and Dentistry of New Jersey, Piscataway, N.J., USA
}

\section{Key Words}

Type I collagen · Nucleation · Apatite $\cdot$ Vertebrate mineralization

\begin{abstract}
The possible means by which type I collagen may mediate mineralization in normal vertebrate bone, tendon, dentin and cementum as well as in pathological mineral formation are not fully understood. One consideration in this regard is that the structure of the protein is somehow important in binding calcium and phosphate ions in a stereochemical configuration conducive to nucleation of apatite crystals. In the present study, type I collagen, packed in a quarter-staggered arrangement in two dimensions and a quasi-hexagonal model of microfibrillar assembly in three dimensions, has been examined in terms of several of its charged amino acid residues. These included glutamic and aspartic acid, lysine, arginine, hydroxylysine and histidine, whose positions along the three $\alpha$-chain axes of the collagen molecule were determined with respect to each other. It was found that the locations of these residues specified sites uniquely suited as potential apatite nucleation centers following binding of calcium and phosphate ions. From this analysis, it would appear that type I collagen provides a template of charged amino acid residues that dictates ion binding critical to subsequent nucleation events for mineral formation in vertebrate tissues.

Copyright $\odot 2008$ S. Karger AG, Basel
\end{abstract}

\section{KARGER}

() 2008 S. Karger AG, Basel

Fax +41613061234

E-Mail karger@karger.ch

www.karger.com
Accessible online at: www.karger.com/cto

\section{Introduction}

Type I collagen is a principal extracellular structural protein in vertebrates that mediates deposition of the mineral, apatite, in normal bone, calcifying tendon, dentin and cementum as well as in numerous examples of pathological calcification. The precise mechanism of mineral formation by type I collagen remains undefined, however. Several lines of evidence, both in vitro and in vivo, support the importance of type I collagen in the onset and progression of mineralization. For example, classical studies of collagen molecules in metastable calcium phosphate solutions demonstrate that normal collagen-mineral interactions occur only if the protein assumes its native structure [Schmitt, 1956; Glimcher and Krane, 1968], and conventional electron microscopy of mineralizing vertebrate tissues shows an intimate and specific structural association between type I collagen and early forming apatite crystals [Robinson, 1952; Robinson and Watson, 1952; Glimcher and Krane, 1968]. The charge distribution of the composite peptide chains of type I collagen can be correlated with a characteristic

Abbreviation used in this paper

$3 \mathrm{D}$

3 dimensions
Dr. William J. Landis

Department of Integrative Medical Sciences

Northeastern Ohio Universities Colleges of Medicine and Pharmacy, Room C-126 4209 State Route 44, Rootstown, OH 44272 (USA)

Tel. +1 330325 6685, Fax +1 330325 5925, E-Mail wjl@neoucom.edu 
Fig. 1. Two-dimensional molecular quarter-staggered packing pattern of a type I collagen microfibril. Each of the five (1-5) molecular segments of a microfibril is composed of $2 \alpha 1(\mathrm{I})$ and $1 \alpha 2$ (I) chains and is shifted by a distance (D) of $67 \mathrm{~nm}$ (in tendon). D consists of a hole (approx. 0.6 D) and overlap (approx. 0.4 D) zone composed of several alternating light and dark bands observed by electron microscopy on staining a microfibril. Staining reflects the underlying amino acid residues in the collagen chains. The diagram is adapted from Silver et al. [2001].

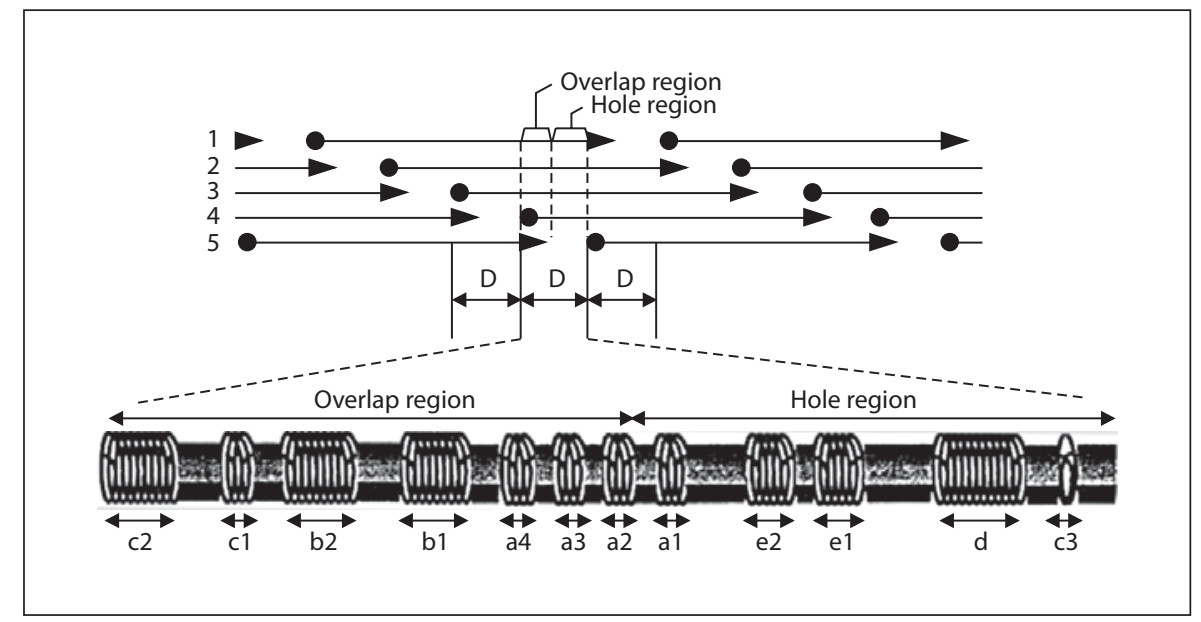

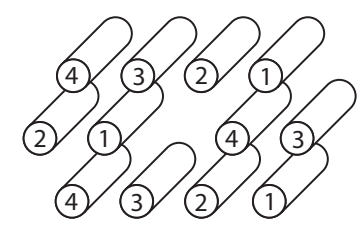

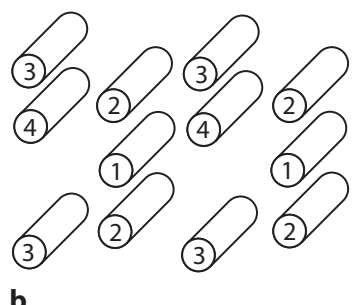

Fig. 2. Three-dimensional schematic of the packing pattern of type I collagen molecules viewed transversely through several hole zone regions. In either the model by Hulmes and Miller [1979] (a) or Orgel et al. [2001] (b), the numbers represent the multiple D unit relative stagger of each collagen molecular segment with respect to its neighbors. The space or distance of separation between amino acid side chains of neighboring segments is reported to be approximately $0.24 \mathrm{~nm}$ and the diameter of a collagen molecule is approximately $1.23 \mathrm{~nm}$ [Lees, 1987].

microscopic pattern of positive staining of the protein, a result leading to the possibility that such charged sites serve as centers for apatite formation [Chapman, 1974; Chapman and Hardcastle, 1974]. More recent high-voltage electron microscopy and three-dimensional (tomographic) imaging [McEwen et al., 1992; Landis et al., 1993] indicate that apatite crystals are initially localized within the so-called hole and overlap regions proposed as critical structural units in the assembly of type I collagen molecules [Hodge and Petruska, 1963; Hodge, 1989]. Once nucleated, the crystals grow into the shape of small platelets [Weiner and Price, 1986; Weiner and
Traub, 1989; Landis et al., 1993] and preferentially increase in their crystallographic $c$-axis length along and parallel to the long axis of collagen associated with them [Glimcher and Krane, 1968]. With further time of development, crystals in neighboring collagen fibrils become coplanar in orientation with their individual $c$-axes parallel to each other [Landis et al., 1993]. All these events and features of crystal nucleation, growth and development, size, shape, orientation and alignment would seem to be influenced by type I collagen, more specifically, its stereochemical nature in or near the hole and overlap zones of the protein [Landis et al., 1993; Landis and Silver, 2002; Landis et al., 2006]. In this circumstance, an analysis was undertaken of the primary, secondary and tertiary structure of type I collagen to gain additional insight into the presence of possible binding sites of calcium and phosphate ions and potential centers for apatite nucleation.

\section{Methods}

The primary amino acid sequence comprising the well accepted molecular character of the human $\alpha 1$ (I) and $\alpha 2$ (I) chains for type I collagen was obtained from the NIH website www.ncbi. nlm.gov/entrez/viewer.fcgi with a search on NP_000079. Molecules were modeled in two dimensions by packing five single collagen triple helical units in the quarter-staggered arrangement described by Hodge and Petruska [1963] (fig. 1) and by assembling them in three dimensions (3D) in the quasi-hexagonal models of microfibrils proposed by either Hulmes and Miller [1979] (fig. 2a) or Orgel et al. [2001] (fig. 2b). Common sites of charged amino acids (glutamic and aspartic acid, lysine, arginine, hydroxylysine and histidine) of type I collagen were identified and their locations determined in relation to one another in the two 
microfibril models. Particular attention was given to the amino acid residues comprising the hole and overlap zones of the type I collagen molecule.

\section{Results}

On careful sequence analysis, the respective locations of the six charged amino acid residues given above were found notable in several two-dimensional features within a single type I collagen molecular segment (triple helical chain) as well as in adjacent segments. First, there are several sites in which the same amino acid is adjacent in each of the three $\alpha$-chains of a single collagen molecule. Second, numerous sites exist in which two of the same amino acids and another of the same charge are adjacent in the three $\alpha$-chains. Third, the same two or three glutamic and/or aspartic acid residues appear close to their counterparts of like charge along $\alpha$-chains. Fourth, many sites can be found in which the same two or three amino acids of one charge lie close to the same two or three amino acids of opposite charge (glutamic acid and lysine or arginine residues; aspartic acid and lysine or arginine residues). Fifth, there are numerous sites in which hydroxylysine contributes charged groups in place of one of the three lysine or arginine residues common in adjacent collagen $\alpha$-chains. As a selected example of some of the first of these features, residue 110 is identically glutamic acid in the three neighboring $\alpha$-chains, and three aspartic acid, arginine or lysine residues are likewise common in their respective $\alpha$-chain locations. Residues 53/54 (aspartic acid and glutamic acid) and 287/288 (both glutamic acid) are illustrative of the fact that these particular amino acids of like charge can frequently be found in close proximity. In addition, residues $554 / 555,566 / 567$ and 791/792 are three of several sites in which amino acids of one charge (glutamic acid or aspartic acid) are present near amino acids of opposite charge (lysine or arginine). The remarkably repetitive and close two-dimensional nature of the six specific charged amino acid residues becomes even more striking when they are represented in the three-dimensional collagen microfibril model. In this instance, many of the locations of these particular amino acids occupying known sites within the type I collagen hole or overlap zones defined by Hodge and Petruska [1963] describe stereochemical configurations that would bind calcium and phosphate ions and likely provide sufficient closeness to induce ion interaction and formation of apatite nucleation centers. In the Hulmes and Miller [1979] quasi-hexagonal model

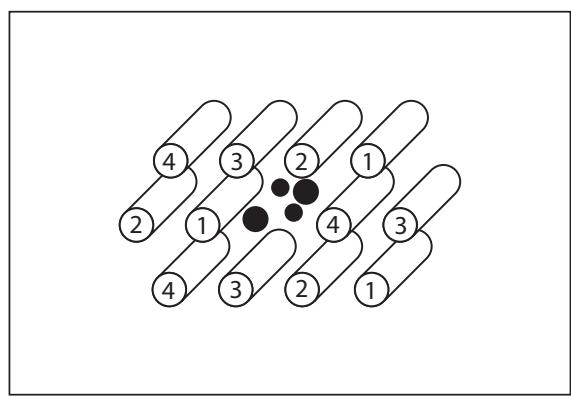

Fig. 3. The diagram shown in figure 2 a but with conceptual localization of two calcium (small filled circles) and two phosphate (large filled circles) ions in a hole zone region of the type I collagen microfibrillar model proposed by Hulmes and Miller [1979]. Ion sizes and positions are not specific and shown only as approximations.

in the vicinity of the hole zone [Hodge and Petruska, 1963; Hodge, 1989], for example, lysine at residue 108 and glutamic acid at residue 110 in collagen segment 1 together with glutamic acid at residue 815 and arginine at residue 816 in segment 4 (adjacent to segment 1) create a pocket that could accommodate calcium and phosphate ions in close proximity (fig. 3). Ion binding may also be enhanced in this same hole zone region through interactions with glutamic acid at residue 116 (segment 1), glutamic acid/arginine at residues $815 / 816$ (segment 4), arginine at residue 350 (segment 2) and lysine and arginine/aspartic acid at residues 581/582 (segment 3). There are instances of numerous additional residues on adjacent collagen segments that are candidate binding sites in either the hole or overlap zones of the models by Hulmes and Miller [1979] or Orgel et al. [2001]. Thus, stereochemical configurations of constituent amino acid residues are present within and between collagen molecular segments of the models that would appear highly favorable to calcium and phosphate ion binding and that might in turn result in the direct formation of apatite nucleation centers.

\section{Discussion}

Collagen is widely recognized as mediating apatite formation in normally mineralizing vertebrate tissues other than enamel and in many instances of pathological or in vitro mineralization. The precise nature of collagenapatite interaction has not been completely understood, however. In this context, some studies in vitro report that collagen alone fails to bind calcium or phosphate ions in 
significant number and does not mineralize [Stetler-Stevenson and Veis, 1986, 1987]. Further, roles in mineral formation have been proposed for several noncollagenous phosphoproteins and other extracellular matrix components that may associate with collagen and augment collagen-mineral interaction or bind mineral ions themselves [Curley-Joseph and Veis, 1979; Stetler-Stevenson and Veis, 1986, 1987; George et al., 1993; MacDougall et al., 1997; Dahl et al., 1998; Ganss et al., 1999; Qin et al., 2003]. In addition, the highly complex extracellular matrices of which collagen is a part in mineralizing vertebrate tissues will most likely limit diffusion of ion clusters and macromolecules to this protein in vivo. In this instance, among several other questions, it is difficult to determine the means of mineral ion access to collagen and whether collagen surface-mediated interactions with calcium and phosphate ions contribute to apatite nucleation events [Landis et al., 1996]. These uncertainties notwithstanding, on the basis of collagen studies modeling its assembly and mineralization in 3D by high-voltage electron microscopic tomography, the suggestion has been made that collagen stereochemistry, that is, the composition and location of its specific amino acid residues and charges, may be critical in directing apatite nucleation, crystal size and shape, orientation and alignment associated with the protein [McEwen et al., 1992; Landis et al., 1993, 1996; Landis and Silver, 2002]. The work reported here presents considerations of type I collagen primary, secondary and tertiary structure and stereochemistry in terms of their potential to mediate nucle- ation events in a microfibrillar model of the protein. Results of this investigation for the first time identify in $3 \mathrm{D}$ a number of type I collagen sites, alone or in close proximity and putative interaction with neighboring sites, that have properties permissive to calcium and phosphate ion binding and their potential nucleation. Such data are important conceptually, regarding not only nucleation but also further events of mineral growth and development in 3D. In a more general conclusion, the data support the view that both two- and three-dimensional structural and stereochemical templates provided by type I collagen are necessary for calcium and phosphate ion binding and subsequent apatite nucleation, growth and development. While the possible roles for other proteins and extracellular constituents of vertebrate tissues, as well as for ion and macromolecular diffusion, in these same events remain to be clarified, type I collagen alone conceptually provides a three-dimensional molecular framework for direct apatite formation. This finding is fundamental to understanding more clearly the basic mechanisms of vertebrate mineralization.

\section{Acknowledgments}

The authors thank Ms. Robin Jacquet from the Department of Integrative Medical Sciences, Northeastern Ohio Universities Colleges of Medicine and Pharmacy, for her assistance with computer graphics. This work was supported by grant AR41452 from the National Institutes of Health (to W.J.L.).

\section{References}

Chapman, J.A. (1974) The staining pattern of collagen fibrils. I. An analysis of electron micrographs. Connect Tissue Res 2: 137-150.

-Chapman, J.A., R.A. Hardcastle (1974) The staining pattern of collagen fibrils. II. A comparison with patterns computer-generated from the amino acid sequence. Connect Tissue Res 2: 151-159.

Curley-Joseph, J., A. Veis (1979) The nature of covalent complexes of phosphoproteins with collagen in the bovine dentin matrix. J Dent Res 58: 1625-1633.

Dahl, T., B. Sabsay, A. Veis (1998) Type I collagen-phosphophoryn interactions: Specificity of the monomer-monomer binding. J Struct Biol 123: 162-168.

-Ganss, B., R.H. Kim, J. Sodek (1999) Bone sialoprotein. Crit Rev Oral Biol Med 10: 79-98.
George, A., B. Sabsay, P.A. Simonian, A. Veis (1993) Characterization of a novel dentin matrix acidic phosphoprotein. Implications for induction of biomineralization. J Biol Chem 268: 12624-12630.

Glimcher, M.J., S.M. Krane (1968) The organization and structure of bone, and the mechanism of calcification; in Ramachandran, G.N., B.S. Gould (eds): Biology of Collagen. Treatise on Collagen. New York, Academic Press, vol IIB, pp 68-251.

-Hodge, A.J. (1989) Molecular models illustrating the possible distribution of 'holes' in simple systematically staggered arrays of type I collagen molecules in native-type fibrils. Connect Tissue Res 21: 137-147.
Hodge, A.J., J.A. Petruska (1963) Recent studies with the electron microscope on ordered aggregates of the tropocollagen macromolecule; in Ramachandran, G.N. (ed): Aspects of Protein Structure. New York, Academic Press, pp 289-300.

-Hulmes, D.J.S., A. Miller (1979) Quasi-hexagonal packing in collagen fibrils. Nature 282: 878-880.

Landis, W.J., K.J. Hodgens, M.J. Song, J. Arena, S. Kiyonaga, M. Marko, C. Owen, B.F. McEwen (1996) Mineralization of collagen occurs on fibril surfaces: evidence from conventional and high voltage electron microscopy and three-dimensional imaging. J Struct Biol 117: 24-35. 
Landis, W.J., F.H. Silver (2002) The structure and function of normally mineralizing avian tendons. Comp Biochem Physiol A 133: 1135-1157.

Landis, W.J., F.H. Silver, J.W. Freeman (2006) Collagen as a scaffold for biomimetic mineralization of vertebrate tissues. J Mater Chem 16: 1495-1503.

Landis, W.J., M.J. Song, A. Leith, L. McEwen, B. McEwen (1993) Mineral and organic matrix interaction in normally calcifying tendon visualized in three dimensions by high voltage electron microscopic tomography and graphic image reconstruction. J Struct Biol 110: 39-54.

Lees, S. (1987) Considerations regarding the structure of the mammalian mineralized osteoid from viewpoint of the generalized packing model. Connect Tissue Res 16: 281303.

MacDougall, M., D. Simmons, X. Luan, J. Nydegger, J. Feng, T.T. Gu (1997) Dentin phosphoprotein and dentin sialoprotein are cleavage products expressed from a single transcript coded by a gene on human chromosome 4: dentin phosphoprotein DNA sequence determination. J Biol Chem 272: 835-842.
McEwen, B.F., M.J. Song, W.J. Landis (1992) Quantitative determination of the mineral distribution in different collagen zones of calcifying tendon using high voltage electron microscopic tomography. J Comput Assist Microsc 3: 201-210.

Orgel, J.P., A. Miller, T.C. Irving, R.F. Fischetti, A.P. Hammersley, T.J. Wess (2001) The in situ supermolecular structure of type I collagen. Structure 9: 1061-1069.

Qin, C., J.C. Brunn, O. Baba, J.N. Wygant, B.W. McIntyre, W.T. Butler (2003) Dentin sialoprotein isoforms: Detection and characterization of a high molecular weight dentin sialoprotein. Eur J Oral Sci 111: 235-242.

Robinson, R.A. (1952) An electron-microscope study of the crystalline inorganic component of bone and its relationship to the organic matrix. J Bone Joint Surg 34: 389434.
Robinson, R.A., M.L. Watson (1952) Collagencrystal relationships in bone as seen in the electron microscope. Anat Rec 114: $383-$ 410.

Schmitt, F.O. (1956) Macromolecular interaction patterns in biological systems. Proc Am Phil Soc 100: 476-486.

Silver, F.H., J.W. Freeman, I. Horvath, W.J. Landis (2001) Molecular basis for elastic energy storage in mineralized tendon. Biomacromolecules 2: 750-756.

Stetler-Stevenson, W.G., A. Veis (1986) Type I collagen shows a specific binding affinity for bovine dentin phosphophoryn. Calcif Tissue Int 38: 135-141.

Stetler-Stevenson, W.G., A. Veis (1987) Bovine dentin phosphophoryn: Calcium ion binding properties of a high molecular weight preparation. Calcif Tissue Int 40: 97-102.

Weiner, S., P.A. Price (1986) Disaggregation of bone into crystals. Calcif Tissue Int 39: $365-$ 375 .

Weiner, S., W. Traub (1989) Crystal size and organization in bone. Connect Tissue Res 21: 259-265. 